Universidad

:

BIBLIOTECA

Document downloaded from the institutional repository of the University of Alcala: https://ebuah.uah.es/dspace/

This is a postprint version of the following published document:

Zapata, F. et al., 2016. Progressing the analysis of Improvised Explosive Devices: Comparative study for trace detection of explosive residues in handprints by Raman spectroscopy and liquid chromatography. Talanta, 161, pp.219-227.

Available at https://doi.org/10.1016/i.talanta.2016.05.057

(C) 2016 Elsevier

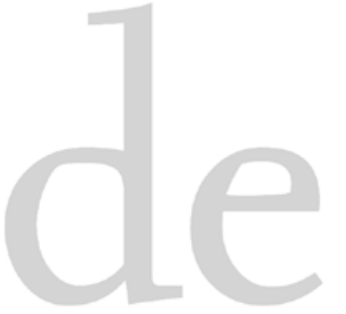

(Article begins on next page)

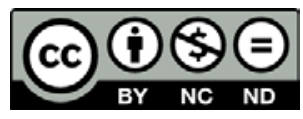

This work is licensed under a

Creative Commons Attribution-NonCommercial-NoDerivatives

4.0 International License. 


\section{Progressing the analysis of Improvised Explosive Devices: Comparative study for trace detection of explosive residues in handprints by Raman spectroscopy and liquid chromatography}

Félix Zapata ${ }^{\mathrm{a}}, \mathrm{M}^{\mathrm{a}}$ Ángeles Fernández de la Ossa ${ }^{\mathrm{a}}$, Elizabeth Gilchrist ${ }^{\mathrm{b}, \mathrm{c}}$, Leon Barron ${ }^{\mathrm{b}}$ Carmen García Ruiz ${ }^{\mathrm{a}, *}$

a Inquifor Research Group, Department of Analytical Chemistry, Physical Chemistry and Chemical Engineering and University Institute of Research in Police Sciences (IUICP), University of Alcalá, Alcalá de Henares (Madrid), Spain. carmen.gruiz@uah.es;

${ }^{\mathrm{b}}$ Department of Pharmacy \& Forensic Science, Analytical \& Environmental Science Division, King's College London, United Kingdom.

${ }^{c}$ Department of Chemistry, University College Cork, College Road, Cork, Ireland.

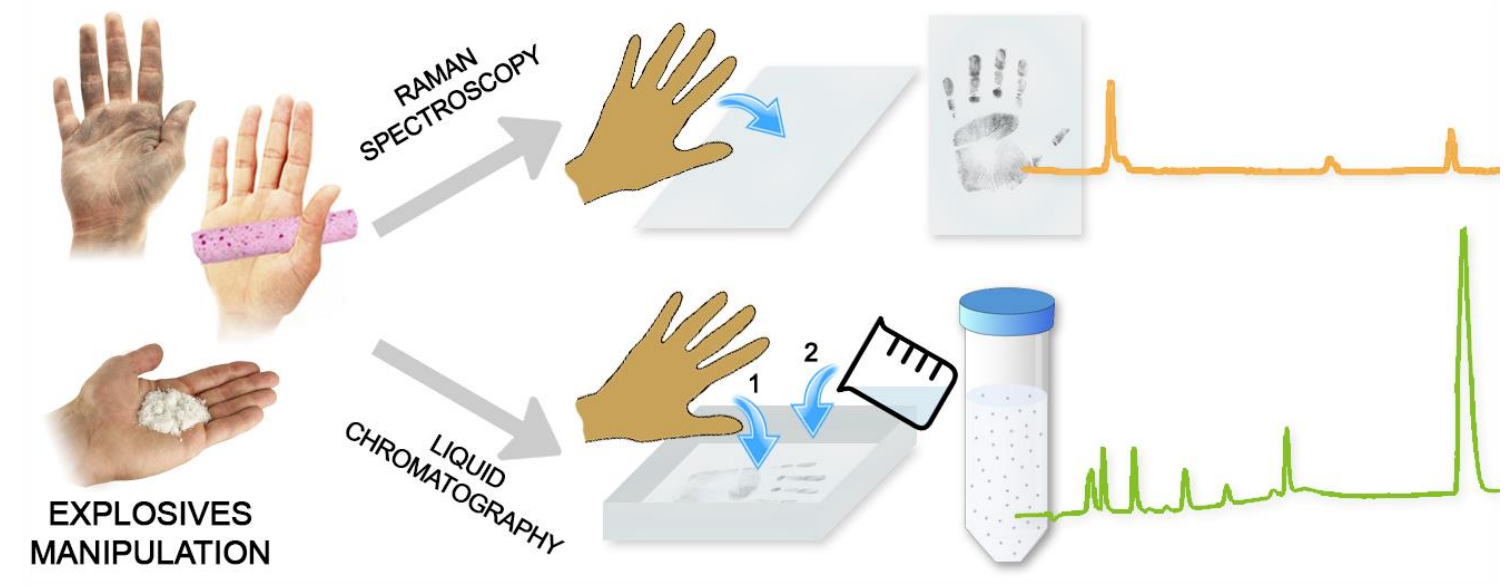

Cite: F. Zapata, M. A. Fernández de la Ossa, E. Gilchrist, L. Barron, C. GarcíaRuiz, Progressing the analysis of Improvised Explosive Devices: Comparative study for trace detection of explosive residues in handprints by Raman spectroscopy and liquid chromatography, Talanta 161 (2016) 219-227. DOI: 10.1016/j.talanta.2016.05.057

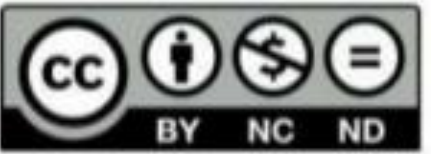




\begin{abstract}
Concerning the dreadful global threat of terrorist attacks, the detection of explosive residues in biological traces and marks is a current need in both forensics and homeland security. This study examines the potential of Raman microscopy in comparison to liquid chromatography (ion chromatography (IC) and reversed-phase high performance liquid chromatography (RP-HPLC)) to detect, identify and quantify residues in human handmarks of explosives and energetic salts commonly used to manufacture Improvised Explosive Devices (IEDs) including dynamite, ammonium nitrate, single- and doublesmokeless gunpowders and black powder. Dynamite, ammonium nitrate and black powder were detected through the identification of the energetic salts by Raman spectroscopy, their respective anions by IC, and organic components by RP-HPLC. Smokeless gunpowders were not detected, either by Raman spectroscopy or the two liquid chromatography techniques. Several aspects of handprint collection, sample treatment and a critical comparison of the identification of compounds by both techniques are discussed. Raman microscopy and liquid chromatography were shown to be complementary to one another offering more comprehensive information for trace explosives analysis.
\end{abstract}

Keywords: Improvised explosive devices (IEDs), handprints, black powder, dynamite, Raman spectroscopy, liquid chromatography. 


\section{Introduction}

Terrorist events have increased in frequency during the last two decades and represent an on-going and extremely harmful global threat [1-7]. Terrorist attacks often involve the use of explosives; compounds which have a great destructive power even in small quantities. Currently, there is a growing trend towards using home-made devices called Improvised Explosive Devices (IEDs) [1-7]. Dreadfully, IEDs have been widely used in recent years in different terrorist attacks including those in Madrid (2004), London (2005), Norway (2011), Boston (2013), Santiago de Chile (2014), Paris (2015), Nigeria, Ankara, Brussels and Pakistan (2016) in addition to war zones as Afghanistan, Syria and Iraq where IEDs are constantly used. IEDs are manually manufactured explosive devices, which consist of a variety of elements arranged in a specific way to produce an explosion. Since their destructive effectiveness lies in being unnoticed, IEDs often have varied design and appearance. Thereby, IEDs often evade being neutralized before an explosive attack. IEDs have become an ideal weapon used by terrorists for several reasons, including their low cost, ease of manufacture and subsequent use and difficulty to be detected [1-7]. Therefore, their detection must be tackled as a matter of urgency to avoid such atrocities and guarantee the security of citizens.

Although IEDs can be manufactured from a large variety of explosive materials including military, commercial, or home-made explosives, they usually contain explosive mixtures based on inorganic energetic oxidants including nitrate, chlorate or perchlorate salts [3, 7-9]. In fact, these explosive mixtures are used to manufacture commercial explosives such as dynamites, black powders and other propellants whose purchase is widely available [3]. In addition, the manufacture of home-made mixtures combining any of these oxidant salts (ammonium nitrate, potassium nitrate, potassium chlorate, potassium perchlorate, etc.) with any fuel (petrol, diesel, sugar, charcoal, flour, etc.) is quite simple [3]. In addition, they are even more freely available than commercial explosives. Ammonium nitrate is used as a fertilizer, but in combination with certain fuels may produce explosive compounds such as dynamite, ammonium nitrate/fuel oil (ANFO) and other nitrate-based explosives [3]. Potassium nitrate, besides also being used as a fertilizer, is a component of black powder widely used to manufacture firecrackers, fireworks and any other pyrotechnic devices. In addition, firecrackers and fireworks usually include potassium chlorate, perchlorate and other nitrate salts such as barium or strontium nitrates to get different colours and effects [9]. 
Liquid chromatography, in particular ion chromatography (IC), has been usually employed to identify and quantify energetic salts mainly through the analysis of their characteristic anions (nitrates, chlorates and perchlorates) [10-15]. Complementarily, reversed-phase liquid chromatography (RP-HPLC) is useful for the analysis of organic non-ionic explosives $[16,17]$. Until now, only a few preliminary studies have proved their suitability to determine residues in human fingermarks $[18,19]$. The detection and identification of explosives in human fingermarks is a comprehensive approach that tackles the challenging task of locating the evidence (illicit substance) as well as potentially identifying a suspect (human fingerprint). The challenge of detecting explosives on fingerprints has been explored using different analytical techniques, being mass spectrometry [20, 21], IR [22-30] and Raman spectroscopy [31-34] those most used up to date. The high sensitivity of mass spectrometry and the speed and nondestructiveness of IR and Raman spectroscopy are highly seductive features. With regards to explosives detection on fingerprints by Raman spectroscopy [31-34], it is important to highlight that the explosives investigated included hexogen (RDX) [31-33], octogen (HMX) [31-33], penthrite (PETN) [31-33], 2,4-dinitrotoluene (2,4-DNT) [34], ammonium nitrate [31-33], potassium nitrate [34] and urea nitrate [34]. In addition, Raman imaging mode was used in those studies and solely the fingerprint area was scanned revealing the presence of explosive residues. Nevertheless, scanning large areas is usually time-consuming, being most effective, in certain cases, the analysis of few selected explosive particles by point measures. Raman microscopy is highly useful to this aim, allowing the rapid detection of microscopic residues. In this study, residues of smokeless gunpowder, black powder and dynamite on human handprints, have been analysed due to the high forensic interest of these explosives. In addition, the whole human handprint, instead of a unique fingerprint, was considered with the aim of studying the distribution of the explosive residues adsorbed on the hand and subsequently left on the handprint.

The aim of this work is to study and compare Raman microscopy and liquid chromatography, using both IC and RP-HPLC, for the analysis of explosives residues in handprints. 


\section{Materials and methods}

\subsection{Materials and chemicals}

\subsubsection{Explosive samples}

Smokeless gunpowder, dynamite and ammonium nitrate samples were kindly provided by the Criminalistic Service of Guardia Civil. Two different smokeless gunpowders were used. According to the information included in the official label [35], a single-base gunpowder sample, which was composed by $94 \%$ of nitrocellulose and a double-base gunpowder sample containing $85 \%$ of nitrocellulose and $10 \%$ of dinitrotoluene (DNT) were included in this study. Dynamite was composed by ethylene glycol dinitrate (EGDN), ammonium nitrate, nitrocellulose, dynamite dye, sawdust, calcium carbonate $\left(\mathrm{CaCO}_{3}\right)$, guar gum and plasticizers, with ammonium nitrate and EGDN declared as its major components [36]. A black powder sample was extracted from a commercial firecracker available in the Spanish market. Black powder is technically defined as a mixture of sulphur, charcoal and potassium nitrate. However, the charge of the pyrotechnic device was composed by sulphur, charcoal and potassium perchlorate. Consequently, and strictly speaking, a black powder substitute product where the potassium nitrate has been replaced by potassium perchlorate, was analysed in this study.

\subsubsection{Reagents}

All reagents were of analytical or reagent grade. For Raman spectroscopy, potassium perchlorate, potassium nitrate, potassium chlorate, sodium nitrate and sodium chlorate were purchased from Sigma-Aldrich (St. Louis, MO, USA).

For IC, chloride, nitrite, nitrate, (BDH Chemicals Ltd, Poole, UK), chlorate, perchlorate, cyanate (Sigma-Aldrich, Gillingham, Dorset, UK) were prepared from their sodium salts; sulphate was prepared from its copper salt (BDH Chemicals Ltd, Poole, UK). Acetate (BDH Chemicals Ltd, Poole, UK) and thiocyanate were prepared from their ammonium salts. Formate was prepared from an ammonium solution (SigmaAldrich, Gillingham, Dorset, UK). Lactate, oxalate (BDH Chemicals Ltd, Poole, UK), phthalate, and benzoate (Sigma-Aldrich, Gillingham, Dorset, UK) were prepared from their acids. All stock solutions were prepared to a concentration of $1000 \mathrm{mg} / \mathrm{L}$ and working standards were prepared daily from these using ultrapure water. Eluents were 
prepared using a $50 \% \mathrm{v} / \mathrm{v} \mathrm{NaOH}$ solution in ultrapure water (Sigma-Aldrich, Gillingham, Dorset, UK) for anion-exchange chromatography. For RP-HPLC, EGDN, DNT and nitroglycerin (NG) were purchased from Kinesis (St Neots, Cambridgeshire, UK). All stock solutions were prepared to a concentration of $100 \mathrm{mg} / \mathrm{L}$ and working standards were prepared daily from these using ultrapure water. Mobile phases were prepared using ammonium acetate and methanol (Fisher Scientific, Loughborough, Leicestershire, UK).

All eluents, stocks, standards and samples solutions were prepared using ultrapure water (18.2 M (.cm) delivered from a Millipore Synergy UV ultra-purification system (Millipore, Bedford, MA, USA). Stock standards were kept in the dark at $5{ }^{\circ} \mathrm{C}$ and were re-prepared fortnightly.

\subsection{Sample preparation and handprint collection}

Smokeless gunpowder, dynamite and ammonium nitrate materials were handled without any prior preparation or treatment by seven volunteers (five women and two men). However, black powder had to be extracted from a commercial firecracker. For this, first the fuse was pulled out and then the cartridge was opened with laboratory scissors in such a way that the pyrotechnic charge was collected for handling.

\subsubsection{Procedure for Raman spectroscopy}

Prior to the explosive handling, and in order to study whether sweat or common exogenous components can influence the Raman spectra of handprints, the seven volunteers washed their hands in ultrapure water and left to air dry for 15 min with the aim of regenerating the sweat as proposed by Gilchrist et al. [18]. During this time, participants were not allowed to handle anything to minimise external contamination. Handprints from both hands were then deposited using a light pressure on the adhesive side of a clear self-adhesive vinyl book cover film of $22 \times 38 \mathrm{~cm}$ size. For sample conservation and protection, films were covered with another piece of film of the same size. Additionally (and with the aim of testing the influence of common dirt), the handprints (right and left) of two participants who had not washed their hands for at least $3 \mathrm{~h}$ and had performed normal daily tasks were also collected. Finally, approximately $5 \mathrm{~g}$ of each type of explosive included in this study were handled by the 
volunteers for $10 \mathrm{~min}$. After, volunteers deposited their handprints following the previously explained procedure on a self-adhesive polyvinyl film. Note that before the explosive manipulation, participants cleaned and dried their hands as was first described. Samples containing the denominated "clean", "natural-contaminated" and "explosive-contaminated" handprints were then analysed by Raman spectroscopy.

Smokeless gunpowder, dynamite and ammonium nitrate identification was directly performed by Raman spectroscopy. However, since black powder has graphite in its composition, which has a strong fluorescence signal in Raman spectroscopy, it required some sample preparation for its identification. About $0.5 \mathrm{~g}$ of black powder were dissolved in $1 \mathrm{~mL}$ of ultrapure water and centrifuged at $4000 \mathrm{rpm}$ during $30 \mathrm{~min}$. Three drops of the supernatant were placed on a pre-cleaned microscope slice and the Raman spectra was registered once the solvent was completely evaporated.

\subsubsection{Procedure for liquid chromatography}

In order to determine the transfer of anionic explosive residues into handprints and to compare Raman with liquid chromatography results, a similar procedure previously described for Raman analysis was followed for preparing the liquid chromatography samples. Volunteers also washed their hands, manipulated $5 \mathrm{~g}$ of each type of explosive during $10 \mathrm{~min}$ and, in this case, deposited their handprints on two similar glass trays. One for each hand (left and right). After, $10 \mathrm{~mL}$ of ultrapure water was added to each tray and both trays were transferred to an ultrasonic bath (USC 2100D, VWR International, Leuven, Belgium) for $30 \mathrm{~min}$ at room temperature $\left(22-23^{\circ} \mathrm{C}\right)$. Extracts were collected into polypropylene vials, along with uncontaminated handprints. Samples were stored in the freezer before being fully defrosted for analysis.

For IC, the extract solutions were dispensed into $0.5 \mathrm{~mL}$ polypropylene vials fitted with in-line filters (Thermo Scientific, Sunnyvale, CA, USA) prior to injection. In some cases it was necessary to dilute the samples to allow for quantification with appropriate reference solutions.

Regarding RP-HPLC, contrary to IC, extracts were transferred into glass vials before direct injection, with no dilution necessary.

\subsection{Instrumentation}

\subsubsection{Raman spectroscopy}


Raman spectroscopic measurements were performed in a Thermo Scientific DXR Raman microscope (Waltham, MA, USA). It was equipped with a $532 \mathrm{~nm}$ excitation wavelength and 900 lines $\mathrm{mm}^{-1}$ grating. Control equipment and data acquisition were performed using the Thermo Scientific Omnic for dispersive Raman 8 software (Waltham, MA, USA). Samples were analysed using $5 \mathrm{~mW}$ of laser power and the 10x, 50x or 100x microscope objectives. The spectra acquisition times were 10 spectra of $2 \mathrm{~s}$ for all samples. Due to the observation of fluorescent signals, was necessary to use the confocal pinhole of $50 \mu \mathrm{m}$ size for the measurement of the dynamite samples. However, the slit of $50 \mu \mathrm{m}$ size was adequate for analysing the ammonium nitrate and the black powder samples. Normalization and baseline correction were applied to all the spectra.

\subsubsection{Liquid chromatography}

IC: Suppressed anion-exchange chromatography was performed on a Dionex DX500 system (Thermo Scientific, Sunnyvale, CA, USA), comprising of a GP40 pump and an ED40 conductivity detector fitted with a Dionex AERS 500 (2 mm ID) electrolytic suppressor (current $=50 \mathrm{~mA}$ ). Instrument control and data acquisition were performed using Chromeleon 6.6. The injection volume was $40 \mu \mathrm{L}$. A quaternary ammoniumbased IonPac AS20 (2 x $250 \mathrm{~mm})$ was used with a flow rate of $0.30 \mathrm{~mL} / \mathrm{min}$. In order to separate all species, a hydroxide gradient was employed using two reservoirs of $5 \mathrm{mM}$ and $40 \mathrm{mM} \mathrm{NaOH}$. The optimised gradient profile was as follows: $5 \mathrm{mM}$ hydroxide from 0-3 min; a linear ramp from 3-8.5 $\mathrm{mM}$ hydroxide from 3-8.5 min; another linear ramp from 8.5-21.5 $\mathrm{mM}$ hydroxide from 8.5-16 $\mathrm{min}$; a final linear ramp from 21.5-40 $\mathrm{mM}$ hydroxide from $16-25.5 \mathrm{~min}$ and maintained at $40 \mathrm{mM}$ hydroxide to $28.8 \mathrm{~min}$. Equilibration time was $15.2 \mathrm{~min}$ (total time $=45 \mathrm{~min}$ ).

RP-HPLC: This was performed on an Agilent HP 1100 system (Agilent Technologies, Berkshire, UK) with detection at $210 \mathrm{~nm}$ using a diode array spectrophotometric detector. Instrument control and data acquisition were performed using the Chemstation software (Agilent Technologies, Berkshire, UK). A Waters Sunfire C18 column of dimensions $2.1 \times 150 \mathrm{~mm}, 3.5 \mu \mathrm{m}$ particle size, (Waters, Milford, MA, USA) was used with a flow rate of $0.15 \mathrm{~mL} / \mathrm{min}$. An isocratic separation method was used with a mobile phase of $8 \mathrm{mM}$ ammonium acetate in $60: 40 \mathrm{MeOH}: \mathrm{H}_{2} \mathrm{O}$. The injection volume was $2 \mu \mathrm{L}$. 


\section{Results and discussion}

\subsection{Raman spectroscopy}

Since Raman spectroscopy just allows to measure small spots or slits in the region of $\mu \mathrm{m}$, firstly, the highest concentration of explosive residues area on handprints was evaluated. As black powder was the only coloured sample of all the tested explosives, it was selected to easily visualize the target areas. Figure 1 shows the scanned handprints images obtained with a common office scanner (CLX-3175, Samsung, China) of two different volunteers. Note that the three areas with a higher concentration of residues were the fingerprints, phalanx base and thenar (indicated as rectangles in Figure 1).

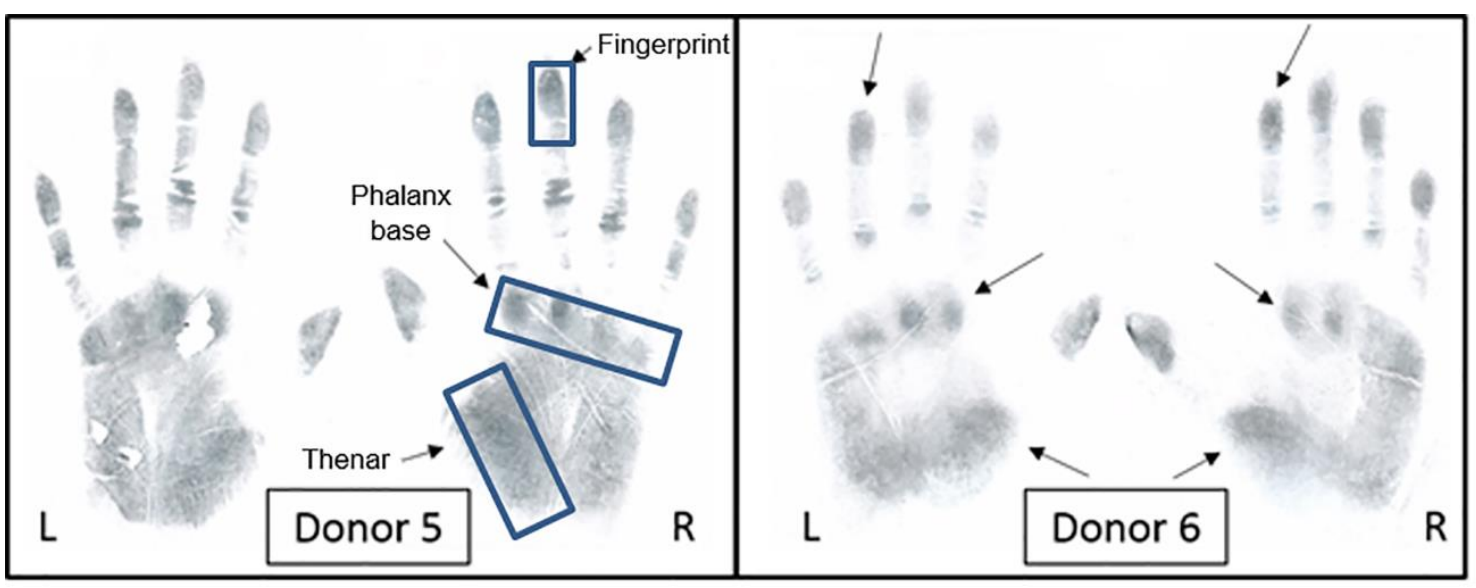

Figure 1. Scanned images of handprints from donor 5 and 6 (L, left and R, right) after the donors had handled black powder. Regions of higher concentration of explosive residues indicated in blue rectangles.

As consequence, for the subsequent Raman analysis only these three areas were considered. Furthermore, using these scanned images the influence of sweat or external contamination on the handprints was evaluated. As expected, no differences among the blank (self-adhesive polyvinyl film without handprint), the handprint collected from a cleaned hand and the handprint from a naturally contaminated hand (at least $3 \mathrm{~h}$ performing routine activities and without cleaning) were observed. However, the presence of contamination on the hands of the volunteers affected the amount of explosive residues deposited in their hand palms. It was observed that higher quantity of residues was present on naturally contaminated handprints compared to cleaned hands (see supporting information Figure 1s). 


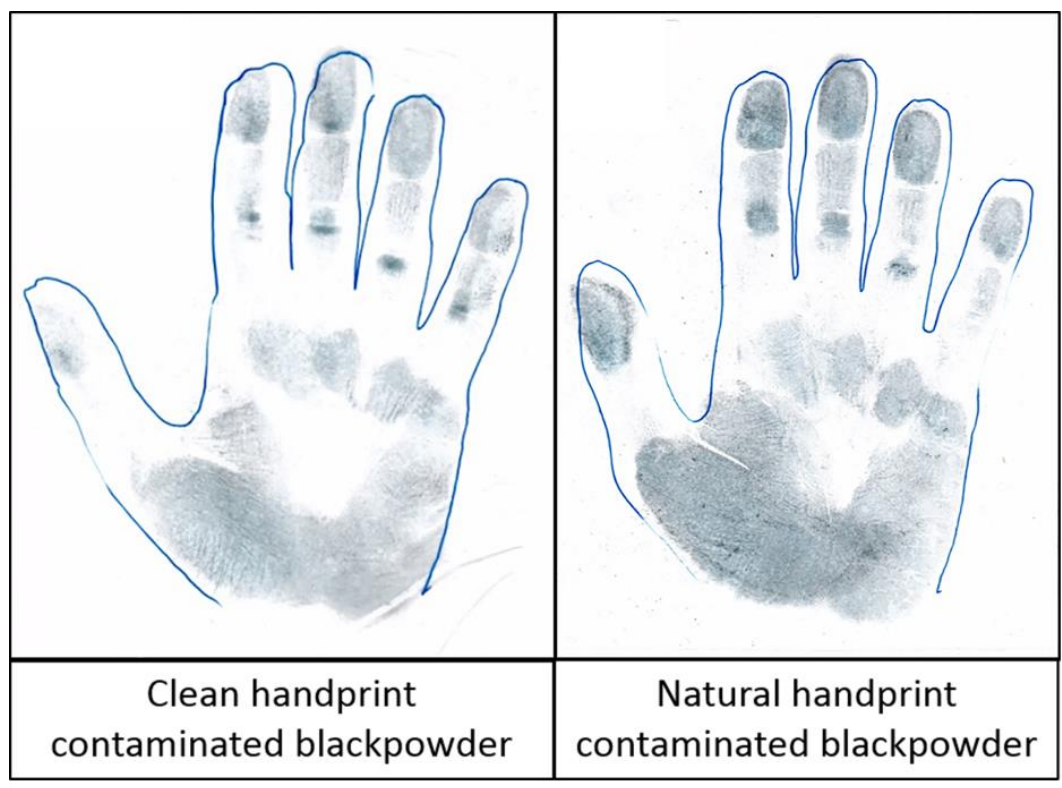

Figure 1s (Supplementary material). Effect of natural contamination on the hands on the amount of explosive residues deposited on handprint. Scanned images of handprints from donor 1 after handling black powder.

To perform the unequivocal identification of each explosive sample and obtain more complete information about the influence of external contamination on the detection of explosive residues, the Raman spectra of blank, clean and natural-contaminated handprints as well as those corresponding to the handling of all explosives were studied. Figure 2 shows the spectra obtained for blanks (self-adhesive polyvinyl film of $5 \times 10$ $\mathrm{cm}$ size), clean and natural handprint samples.

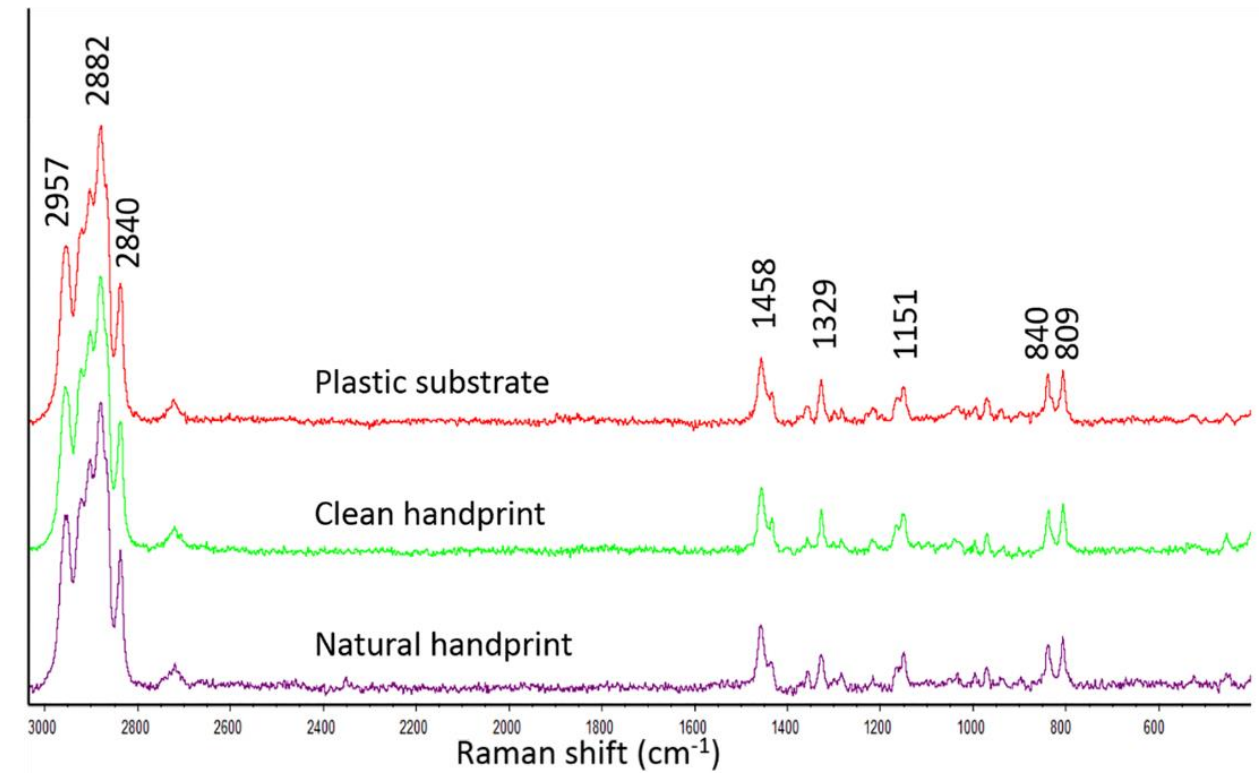

Figure 2. Influence of sweat or dirt in the Raman spectra. Spectral comparison (from top to bottom) of the self-adhesive polyvinyl film as blank, a clean handprint and a natural handprint. Raman conditions: laser at 532, $5.0 \mathrm{~mW}, 10 \mathrm{x}$ magnification objective lens, and confocal pinhole size of $25 \mu \mathrm{m}$. Spectral acquisition times: $2 \mathrm{~s}$ x 10 acquisitions. 
As can be seen, similar spectra were obtained in all three different samples. Although Raman spectroscopy did not allow the detection of natural residues from dirt or sweat, it did enable the direct detection of explosive residues in handprints with little/no detectable interference.

In order to test the efficiency of Raman spectroscopy to detect trace explosive residues on handprints, the handprints (deposited on several self-adhesive polyvinyl films of $22 \mathrm{x}$ $38 \mathrm{~cm}$ size following the methodology indicated in Section 2.2.1) were collected after the handling of single- and double-base smokeless gunpowder, black powder, dynamite and ammonium nitrate samples by seven different participants. Two handprints (from both hands) were analysed per volunteer. Intense fluorescence signals hiding the characteristic signals of the explosive of interest were observed for both types of smokeless gunpowder and black powder samples (results not shown). Several attempts to reduce this excess of fluorescence were made, such as the reduction of the laser power to $3 \mathrm{~mW}$, the time and number of acquisitions, as well as the exchange of the 532 $\mathrm{nm}$ laser for one less powerful of $780 \mathrm{~nm}$, without success. Therefore, smokeless gunpowder samples were excluded from the study by Raman spectroscopy because they presented a very intense fluorescence response to Raman radiation. However, a little sample treatment, as previously mentioned in the method section, was applied to black powder in order to try the identification of any of its components. After complete evaporation, the crystals obtained from the black powder solution were analysed by Raman spectroscopy. Comparing the obtained spectrum with the reference spectra of different inorganic salts (potassium perchlorate, potassium chlorate, sodium chlorate, sodium nitrate, potassium nitrate and ammonium nitrate) commonly present in pyrotechnic charges, it was possible to identify the presence of potassium perchlorate in its composition (see Figure 3). This agrees with the later identification of perchlorate in the extracts from black powder contaminated handprints using IC. In addition, this preliminary study, in which Raman signatures from several salts were compared, seemed to reveal that not only the salts with different anions (nitrate $v s$ chlorate $v s$ perchlorate) could be differentiated by Raman spectroscopy, but also salts based on the same anion and different cation. It is worth comparing, for example, sodium, potassium and ammonium nitrates, whose nitrate bands were not located at the same Raman shift. Bands from sodium nitrate were located at 1383, 1065 and $722 \mathrm{~cm}^{-1}$, whereas bands from potassium nitrate were located at 1357, 1047 and $712 \mathrm{~cm}^{-1}$ and bands from 
ammonium nitrate were located at 1041 and $714 \mathrm{~cm}^{-1}$ (see Figure 3). In fact, by using a Raman spectrometer with an instrumental precision of about $\pm 2 \mathrm{~cm}^{-1}$ (the one that our Raman instrument had) even potassium nitrate can be distinguished from ammonium nitrate according to the $6 \mathrm{~cm}^{-1}$ shift between their bands at 1047 and $1041 \mathrm{~cm}^{-1}$, respectively. The cation seems to affect the polarizability of the anion and therefore there are slight differences in the Raman shift of the bands of anion depending on the respective cation.

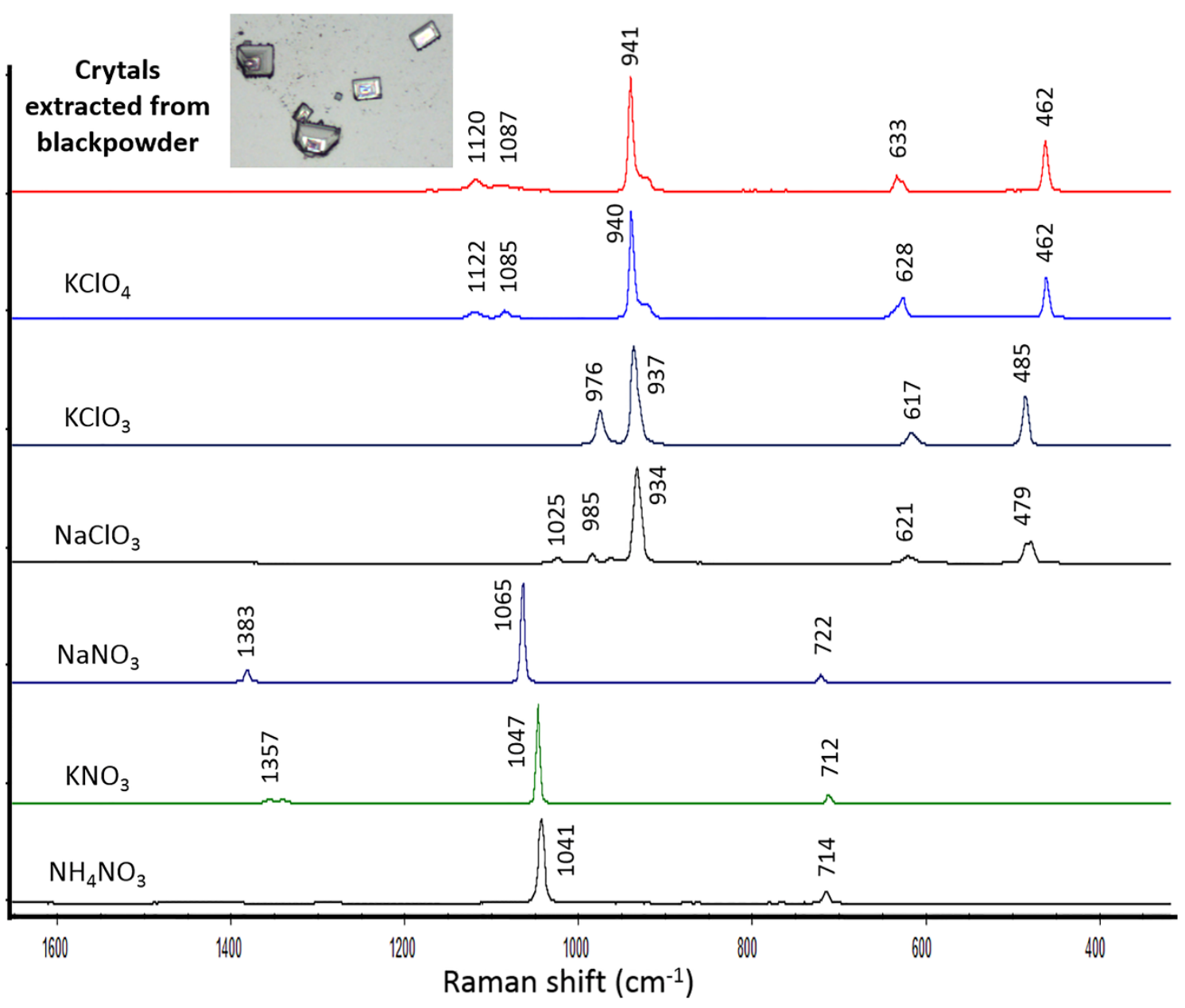

Figure 3. Spectrum of black powder extracted from a commercial firecracker and handled in this work (red) compared to other inorganic salts (potassium perchlorate, potassium chlorate, sodium chlorate, sodium nitrate, potassium nitrate and ammonium nitrate) commonly present in commercial firecrackers. Raman conditions as in Figure 2.

Regarding the handprints contaminated with dynamite and ammonium nitrate, their direct identification was achieved by Raman spectroscopy. Handprint residues were microscopically examined with the aim of searching particles from the explosives, whose Raman spectra allowed their identification by comparison with the Raman spectrum of ammonium nitrate shown in Figure 3. 
It is interesting to highlight that two types of residues from explosives were detected on handprints using the Raman microscope, including large particles from the explosive which almost overlapped an epidermal ridge, and residues of the explosive quite smaller and contained within the fingerprint ridges. The large particles, which were mainly found in those areas of the hand in which there are deep wrinkles and lines due to muscular and joint contractions, mostly ranged between 10 and $30 \mu \mathrm{m}$ in size. This fact is probably explained by the largest and deepest surface in those wrinkles in which large particles may be adhered. These particles provided highly intense and well-defined Raman spectra. The largest particles were between 60 and $100 \mu \mathrm{m}$. No particles larger than $100 \mu \mathrm{m}$ were detected, which probably indicates that so big particles are not properly adhered in the skin and fall down immediately. On the other hand, the small particles found within the fingerprint ridges, which are the most abundant residues in latent fingerprints, provided also well-defined but less intense Raman spectra. In fact, these small particles were analysed using $50 \mathrm{x}$ or $100 \mathrm{x}$ magnification objectives since their size was below $5 \mu \mathrm{m}$. As previously reported by Verkouteren [24] for hexogen (RDX) and penthrite (PETN) residues, particles smaller than $10 \mu \mathrm{m}$ have the highest frequency in fingerprints. Analogously, the largest particles reported by Verkouteren in fingerprints [24] ranged from 50 to $100 \mu \mathrm{m}$.

Figure 4 shows three examples of the spectra obtained for three different particles in both dynamite (a) and ammonium nitrate (b) contaminated handprints, as well as the spectra registered for the standard sample of dynamite or ammonium nitrate and the self-adhesive polyvinyl film. Spectra registered for the handprint generated after the handling of dynamite corresponded to ammonium nitrate (bands at 1041 and $714 \mathrm{~cm}^{-1}$ ) and self-adhesive polyvinyl film (see Figure 2: bands at 2957, 2882, 2840, 1458, 1329, $\left.1151,840,809 \mathrm{~cm}^{-1}\right)$. In the case of the handprints deposited after handling ammonium nitrate, these were identical to those registered for the dynamite handprints. This fact was explained through the sample composition, since the main components of dynamite are ammonium nitrate and EGDN. However, EGDN has a high vapour pressure $(7.0 \mathrm{x}$ $10^{-2}$ torr at $25^{\circ} \mathrm{C}$ ) and is quickly volatilized at room conditions, used in this research, making its detection difficult after 10 min of dynamite handling and leaving essentially only ammonium nitrate residues on the handprint. 

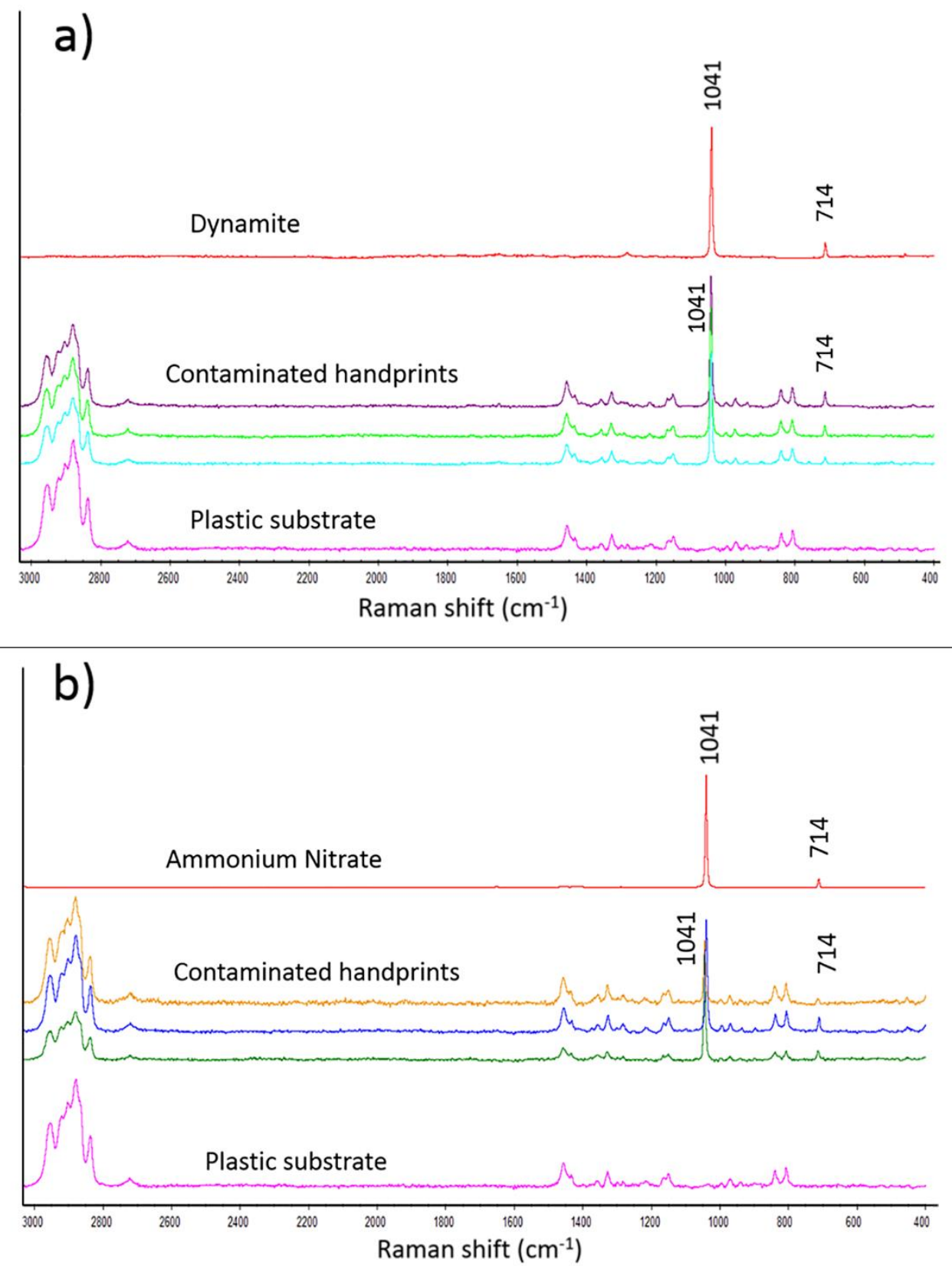

Figure 4. Dynamite and ammonium nitrate identification in handprints. Spectral comparison of (a) standard dynamite, three different handprints from donors after handling dynamite and the substrate and (b) standard ammonium nitrate, handprints from three donors after handling ammonium nitrate and the substrate. Raman conditions as in Figure 2.

Finally, the mass of the particles detected by Raman microscopy was estimated as follows. Assuming a spherical particle with a diameter of $10 \mu \mathrm{m}$, its volume is 523.6 $\mu \mathrm{m}^{3}$ (assuming a cubic particle its volume would be $1000 \mu \mathrm{m}^{3}$ ). In the knowledge that ammonium nitrate has a density of $1.725 \mathrm{~g} / \mathrm{cm}^{3}$, the mass of some of the particles analysed by Raman was $0.9 \mathrm{ng}$ (1.7 ng if we assume cubic particles). Moreover, those particles smaller than $5 \mu \mathrm{m}$ in diameter which were also analysed and identified by Raman weighed less than $0.1 \mathrm{ng}$. 


\subsection{Liquid chromatography}

Using IC, the analysis of uncontaminated extracts (from "clean" and "naturalcontaminated" latent handprints) revealed low $\mu \mathrm{g} / \mathrm{mL}$ concentrations of lactate, acetate, formate, chloride nitrite, nitrate and sulphate in agreement with previous work [18]. Oxalate (for $n=2$ donors) and thiocyanate ( $n=1$ donor) were found at low $\mu \mathrm{g} / \mathrm{mL}$ quantities, below the limit of quantitation (LOQ) [19].

The extracts obtained from the latent handprints deposited after handling single- and double-base smokeless gunpowders contained no characteristic anions. There was also no noticeable change in the concentrations of anions present in the control handprints, including nitrite and nitrate.

Using RP-HPLC, the analysis of these extracts showed no traces of nitrocellulose in any of the samples $(n=5)$. This is not wholly surprising as nitrocellulose, which is the main component in both smokeless gunpowders, is not soluble in water and is therefore itself unlikely to have extracted into solution. DNT, which was supposedly present in the double-base gunpowder, was also not detected. This is likely due to water being an unsuitable extraction solvent for these particular analytes, or perhaps a more sensitive method utilizing mass spectrometry as the detection mode is required.

Regarding the black powder contaminated handprints, the extracts were only analysed by IC due to the inorganic composition of black powder. The extracts yielded $\mu \mathrm{g} / \mathrm{mL}$ concentrations $(0-11 \mu \mathrm{g} / \mathrm{mL})$ of benzoate as well as perchlorate $(1-172 \mu \mathrm{g} / \mathrm{mL})$ as shown in Figure 5. These analytes have previously been detected in other black powder substitutes, although nitrate concentrations were not found to similarly increase [18]. As this particular IC method was only applied to determine the anionic components of the handprint extracts, the presence of any counter ions, such as potassium were not confirmed in this case. Concentrations of sulphate, despite the firecracker containing sulphur, did not increase appreciably in any of the 7 donors handprint extracts (average increase of $0.15 \mu \mathrm{g} / \mathrm{mL}$ ). However, sulphate typically results from oxidation of sulphur and therefore not expected in pre-blast samples. 


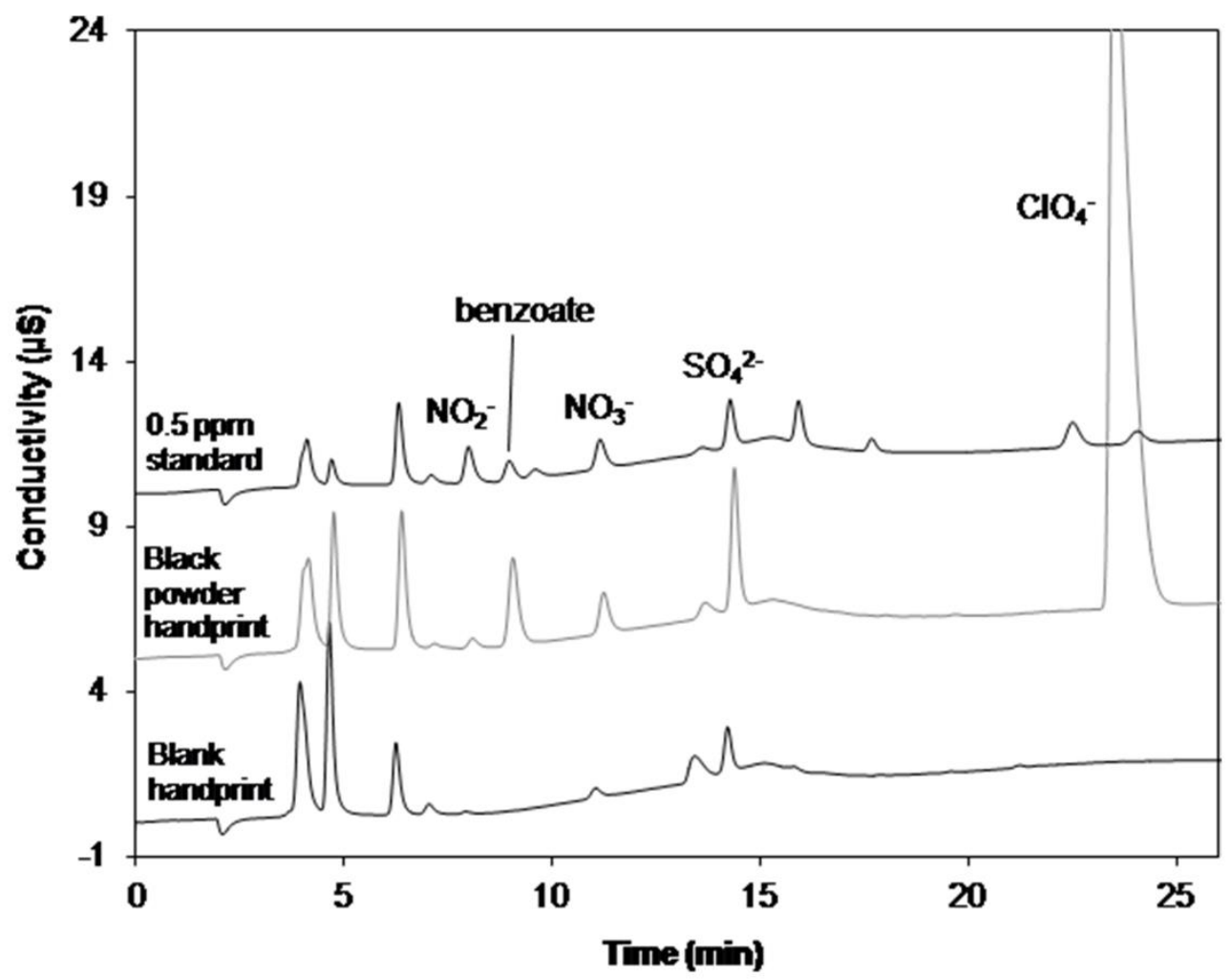

Figure 5. Chromatogram showing an uncontaminated handprint; a handprint after the donor had handled black powder; and $0.5 \mu \mathrm{g} / \mathrm{mL}$ anion standard. IC conditions: Injected volume: $40 \mu \mathrm{L}$, flow rate $0.30 \mathrm{~mL} / \mathrm{min}$, hydroxide gradient: $5 \mathrm{mM}$ from 0-3 $\mathrm{min}$; 3-8.5 mM from 3-8.5 min; 8.5$21.5 \mathrm{mM}$ from $8.5-16 \mathrm{~min} ; 21.5-40 \mathrm{mM}$ from $16-25.5 \mathrm{~min} ; 40 \mathrm{mM}$ to $28.8 \mathrm{~min}$. Chromatograms offset on signal axis by $5 \mu \mathrm{S}$ for clarity.

The extracts from the handprints after handling dynamite and ammonium nitrate both showed greatly increased concentrations of nitrate $(6-266 \mu \mathrm{g} / \mathrm{mL}$ and $2-479 \mu \mathrm{g} / \mathrm{mL}$ respectively) as shown in Figure 6. As the dynamite contained ammonium nitrate, these results were not unexpected. In general, the extracts from the dynamite contaminated handprints contained lower concentrations of nitrate with an average of $95 \mu \mathrm{g} / \mathrm{mL}$ compared to an average measurement of $213 \mu \mathrm{g} / \mathrm{mL}$ in the extracts from handprints after handling the ammonium nitrate sample. There was no noticeable increase in carbonate despite the presence of $\mathrm{CaCO}_{3}$ as a minor component in the dynamite sample, and concentrations of nitrite remained below the method LOQ. 


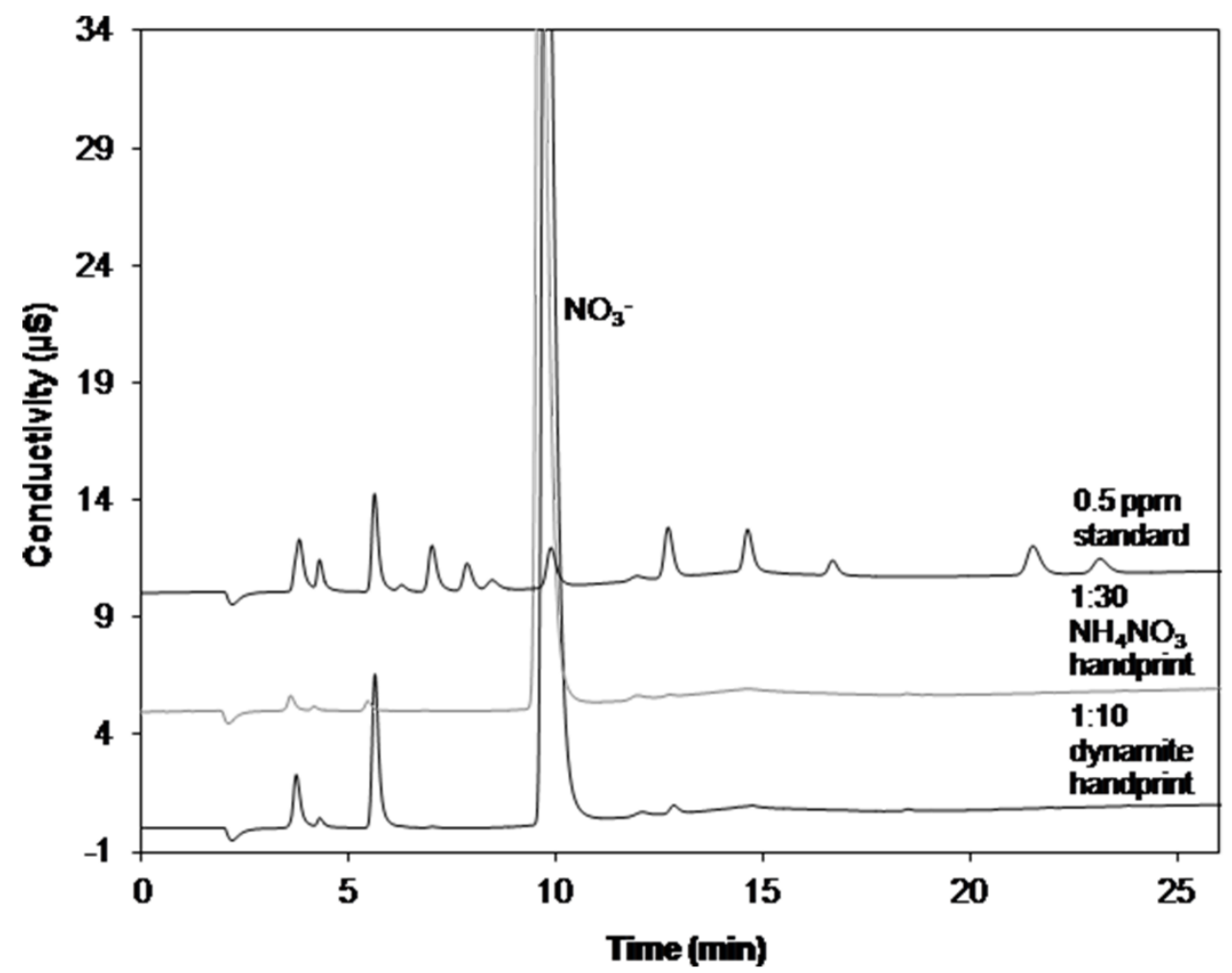

Figure 6. Chromatogram showing a 1:10 dilution of a handprint after the donor had handled dynamite; a 1:30 dilution of a handprint after the donor had handled ammonium nitrate; and 0.5 $\mu \mathrm{g} / \mathrm{mL}$ anion standard. Chromatographic conditions and chromatograms offset as in Figure 5.

The other major component of the dynamite sample was EGDN, which was determined by RP-HPLC. In this case, the chromatograms of the extracts from the handprints left after handling dynamite showed a peak at 4.34 min which matched that of the EGDN standard (4.32 min), as displayed in Figure 7. This was surprising as it was expected that EGDN would be mainly lost to the atmosphere, and any remnants would not extract into an aqueous solution. A peak was also present in at $6.42 \mathrm{~min}$ which matched the retention of NG. However, this was also present in the blank handprint, suggesting it to be a component of sweat rather than NG, although this was not identified. In addition, a system peak was present at $5.05 \mathrm{~min}$ in all samples.

Results obtained for all three sample types analysed here make it apparent that the transfer between the sample, hand and glass surface is highly variable between donors, and there was no correlation to suggest certain donors are particularly better or worse at transferring these residues. 


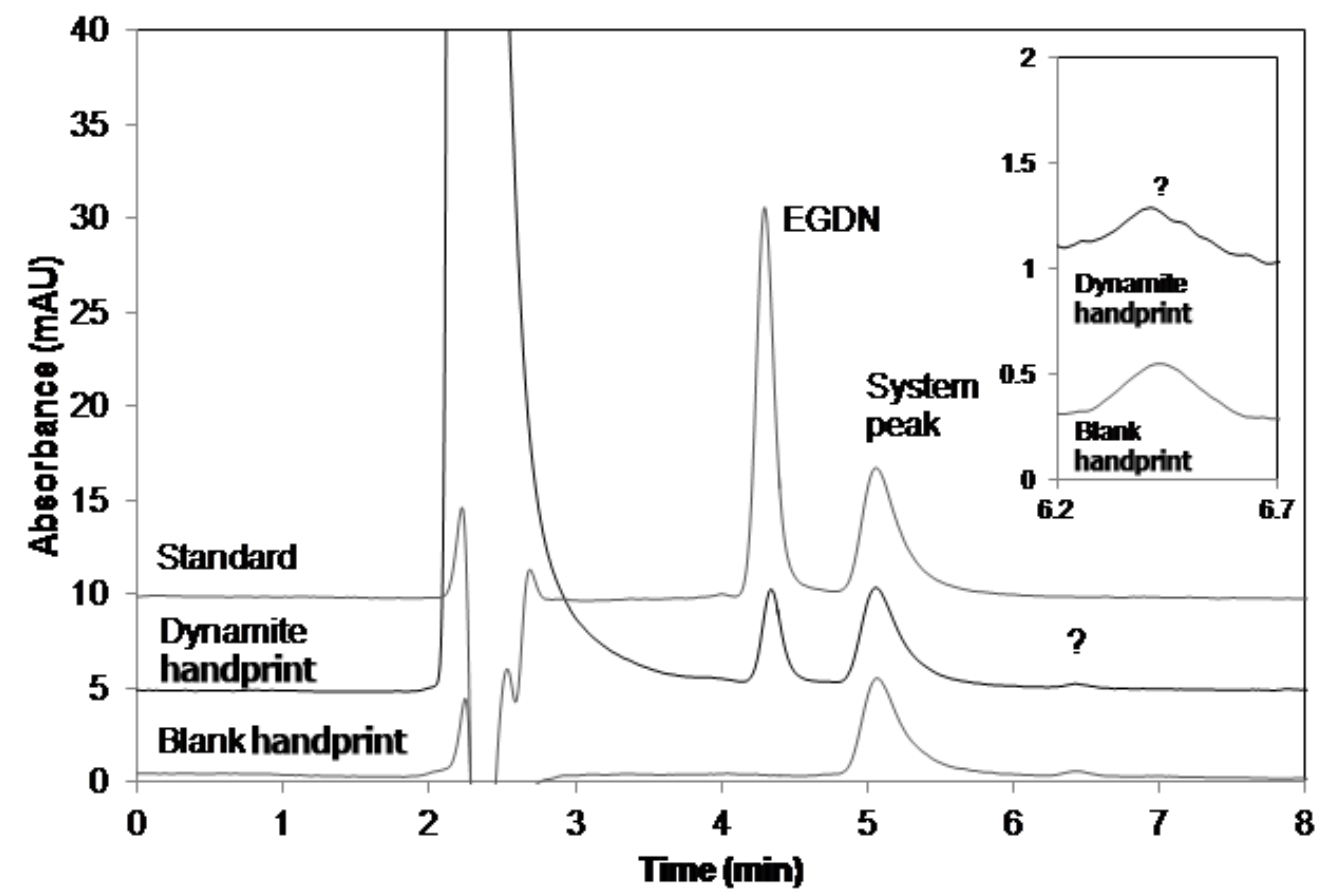

Figure 7. Chromatogram showing a blank handprint; a handprint after the donor had handled dynamite; and $5 \mu \mathrm{g} / \mathrm{mL}$ EGDN standard. RP-HPLC conditions: Injected volume: $2 \mu \mathrm{L}$, flow rate $0.15 \mathrm{~mL} / \mathrm{min}$, isocratic elution with $60: 40(\mathrm{v} / \mathrm{v}) \mathrm{MeOH} / \mathrm{H}_{2} \mathrm{O}$ in $8 \mathrm{mM}$ ammonium acetate. Chromatograms offset on signal axis by $5 \mathrm{mAU}$ for clarity. Insert of the unknown peak at 6.42 min offset by $1 \mathrm{mAU}$.

\subsection{Raman spectroscopy and liquid chromatography comparison}

A comparative study of the techniques revealed that, using Raman spectroscopy, ammonium nitrate was detected in ammonium nitrate and dynamite contaminated human handprints, while using IC, the nitrate anion was detected in both samples, and EGDN was also detected in dynamite extracts through RP-HPLC. Analogously, black powder was detected through the identification of the perchlorate anion by IC and the potassium perchlorate salt by Raman spectroscopy. Single- and double-smokeless gunpowders were undetected either by liquid chromatography, mainly due to the inefficiency of water extracts to dissolve their non-ionic main components such as nitrocellulose, or by Raman spectroscopy due to their fluorescence. Interestingly, Raman spectroscopy, unlike IC, allowed the identification of the salt (anion + cation). However, liquid chromatography allowed quantitative analysis, being especially useful for trace detection. Regarding the current study, quantitative differences in ammonium nitrate concentrations were found by IC between ammonium nitrate and dynamitecontaminated handprints. Ammonium nitrate-contaminated handprints had a higher concentration of nitrate than dynamite contaminated handprints. That may be explained 
according to the dynamite composition, which includes a $60-70 \%$ of ammonium nitrate in its composition. Interestingly, by performing the designed procedure to prepare the samples for liquid chromatography analysis, the residues from the entire handprint were collected and determined. On the contrary, the Raman signal was due to the solid particles of residues located within the laser spot. Therefore, only a small part of the residues in the handprint was examined, being enough to identify the compound, and providing the interesting potential to visualise the exact location of residues located in a whole handprint. Given its non-destructive nature, Raman could be previously used for rapid identification of explosives followed by liquid chromatography (either IC, RPHPLC or a combination depending on the results from the Raman) for cross-validation or quantitative purposes.

\section{Conclusions and future trends}

In conclusion, Raman spectroscopy and liquid chromatography (IC and RP-HPLC) have turned out to be suitable complementary techniques. In fact, in this case Raman spectroscopy has supported the identification of compounds since it was able to identify the salt (anion + cation), whereas IC has supported the quantification of the salt based on the ion concentration. Therefore, both techniques are appropriate to determine the ionic species from explosive residues on handprints and fingerprints. In addition, RPHPLC enabled the determination of EGDN in extracts from dynamite. As consequence of the different information provided by both techniques, we propose residues should be first identified by Raman spectroscopy (since Raman spectroscopy is more rapid in comparison to liquid chromatography) and, then, quantified by liquid chromatography, in the appropriate mode.

As future trend, it is important to mention that Raman spectroscopy seems to allow the discrimination between salts even when they are composed by the same anion due to the effect of the counter cation. Thus, further studies are being performed considering a wider number of salts in order to confirm this capability of Raman spectroscopy and research this phenomenon more thoroughly (i.e. how the cation shifts the anion band, the influence of charge and size of the cation, etc.) 


\section{Acknowledgements}

The authors thank European Commission for the Project HOME/2011/ISEC/AG/4000002480 funded with the financial support of the Prevention of and Fight against Crime Programme from the European Commission - DirectorateGeneral Home Affairs. This publication reflects the views only of the authors, and the European Commission cannot be held responsible for any use which may be made of the information contained therein. The authors also thank the University of Alcala for the research project CGG2013/EXP-021. Félix Zapata thanks the University of Alcalá for his research grant. Elizabeth Gilchrist would like to acknowledge the support of the King's Continuation Scholarship.

The authors have declared no conflicts of interest.

\section{References}

[1] P. R. Laska, Bombs, IEDs, and explosives: Identification, investigation, and disposal techniques, CRC Press Taylor \& Francis Group, Boca Raton, USA, 2016.

[2] J. Yinon, Counterterrorist Detection Techniques of Explosives, Elsevier, Netherlands, 2007.

[3] M. Marshall, J. Oxley, Aspects of Explosives Detection, Elsevier, Netherlands, 2009.

[4] R. S. Golightly, W. E. Doering, M. J. Natan, Surface-enhanced Raman spectroscopy and homeland security: a perfect match? ACS Nano 3 (2009) 2859-2869.

[5] A. Hakonen, P. O. Andersson, M. S. Schmidt, T. Rindzevicius, M. Käll, Explosive and chemical threat detection by surface-enhanced Raman scattering: A review, Anal. Chim. Acta 893 (2015) 1-13.

[6] A. Hakonen, T. Rindzevicius, M. S. Schmidt, P. O. Andersson, L. Juhlin, M. Svedendahl, A. Boisen, M. Käll, Detection of nerve gases using surface-enhanced Raman scattering substrates with high droplet adhesion, Nanoscale 8 (2016) 1305-1308.

[7] M. A. Fernández de la Ossa, J. M. Amigo, C. García-Ruiz, Detection of residues from explosive manipulation by near infrared hyperspectral imaging: A promising forensic tool, Forensic Sci. Int. 242 (2014) 228-235.

[8] N. Nuntawong, P. Eiamchai, S. Limwichean, B. Wong-ek, M. Horprathum, V. Patthanasettakul, A. Leelapojanapom, S. Nakngoenthong, P. Chindaudom, Trace detection of 
perchlorate in industrial-grade emulsion explosive with portable surface-enhanced Raman spectroscopy, Forensic Sci. Int. 233 (2013) 174-178.

[9] C. Martín-Alberca, C. García-Ruiz, Analytical techniques for the analysis of consumer Fireworks, Trends Anal. Chem. 56 (2014) 27-36.

[10] C. Johns, R. A. Shellie, O. G. Potter, J. W. O’Reilly, J. P. Hutchinson, R. M. Guijt, M. C. Breadmore, E. F. Hilder, G. W. Dicinoski, P. R. Haddad, Identification of homemade inorganic explosives by ion chromatographic analysis of post-blast residues, J. Chromatography A 1182 (2008) 205-214.

[11] E. Tyrrell, E. F. Hilder, R. A. Shalliker, G. W. Dicinoski, R. A. Shellie, M. C. Breadmore, C. A. Pohl, P. R. Haddad, Packing procedures for high efficiency, short ion-exchange columns for rapid separation of inorganic anions, J. Chromatography A 1208 (2008) 95-100.

[12] H. Meng, T. Wang, B. Guo, Y. Hashi, C. Guo, J. Lin, Simultaneous determination of inorganic anions and cations in explosive residues by ion chromatography, Talanta 76 (2008) 241-245.

[13] E. Tyrrell, R. A. Shellie, E. F. Hilder, C. A. Pohl, P. R. Haddad, Fast ion chromatography using short anion exchange columns, J. Chromatography A 1216 (2009) 8512-8517.

[14] G. L. Lang, K. M. Boyle, The analysis of black powder substitutes containing ascorbic acid by Ion Chromatography/Mass spectrometry, J. Forensic Sci. 54 (2009) 1315-1322.

[15] L. Barron, E. Gilchrist, Ion chromatography-mass spectrometry: A review of recent technologies and application in forensic and environmental explosives analysis, Anal. Chim. Acta 806 (2014) 27-54.

[16] D. Gaurav, A. K. Malik, P. K. Rai, High-Performance Liquid Chromatographic Methods for the Analysis of Explosives, Critical Reviews Anal. Chem. 37(4) (2007) 227-268.

[17] B. Paull, C. Roux, M. Dawson, P. Doble, Rapid screening of selected organic explosives by high performance liquid chromatography using reversed-phase monolithic columns, J. Forensic Sci. 49(6) (2004) 1181-1186.

[18] E. Gilchrist, N. Smith, L. Barron, Probing gunshot residue, sweat and latent human fingerprints with capillary-scale ion chromatography and suppressed conductivity detection, Analyst 137 (2012) 1576 - 1583.

[19] C. Love, E. Gilchrist, N. Smith, L. Barron, Detection of anionic energetic material residues in enhanced fingermarks on porous and non-porous surfaces using ion chromatography, Forensic Sci. Int. 231 (2013) 150-156. 
[20] P. Hazarika, D. A. Russell, Advances in Fingerprint Analysis, Angew. Chem. Int. 51 (2012) 3524-3531.

[21] F. Rowell, J. Seviour, A. Y. Lim, C. G. Elumbaring-Salazar, J. Loke, J. Ma, Detection of nitro-organic and peroxide explosives in latent fingermarks by DART- and SALDI-TOF-mass spectrometry, Forensic Sci. Int. 221 (2012) 84-91.

[22] P. H. Ng, S. Walker, M. Tahtouh, B. Reedy, Detection of illicit substances in fingerprints by infrared spectral imaging, Anal. Bioanal. Chem. 394 (2009) 2039-2048.

[23] E. V. Bertseva, A. V. Savin, Explosives trace detection in the process of biometrical fingerprint identification for access control, Proc. SPIE 6594 (2007) 65940F/1-65940F/10.

[24] J. R. Verkouteren, Particle characteristics of trace high explosives: RDX and PETN, J. Forensic Sci. 52 (2007) 335-340.

[25] T. Chen, Z. D. Schultz, I. W. Levin, Infrared spectroscopic imaging of latent fingerprints and associated forensic evidence, Analyst 134 (2009) 1902-1904.

[26] R. Bhargava, R. S. Perlman, D. C. Fernandez, I. W. Levin, E. G. Bartick, Non-invasive detection of superimposed latent fingerprints and inter-ridge trace evidence by infrared spectroscopic imaging, Anal. Bioanal. Chem. 394 (2009) 2069-2075.

[27] Y. Mou, J. W. Rabalais, Detection and identification of explosive particles in fingerprints using Attenuated Total Reflection-Fourier Transform Infrared Spectromicroscopy, J. Forensic Sci. 54 (2009) 846-850.

[28] A. Banas, K. Banas, M. B. H. Breese, J. Loke, B. Heng Teo, S. K. Lim, Detection of microscopic particles present as contaminants in latent fingerprints by means of synchrotron radiation-based Fourier transform infra-red micro-imaging, Analyst 137 (2012) 3459-3465.

[29] A. Banas, K. Banas, M. B. H. Breese, J. Loke, S. K. Lim, Spectroscopic detection of exogenous materials in latent fingerprints treated with powders and lifted off with adhesive tapes, Anal. Bioanal. Chem. 406 (2014) 4173-4181.

[30] M. A. Fernández de la Ossa, C. García-Ruiz, J. M. Amigo, Near infrared spectral imaging for the analysis of dynamite residues on human handprints, Talanta 130 (2014) 315-321.

[31] E. D. Emmons, A. Tripathi, J. A. Guicheteau, S. D. Christesen, A. W. Fountain, Raman Chemical Imaging of Explosive-Contaminated Fingerprints, Appl. Spectrosc. 63 (2009) 1197 1203. 
[32] A. Tripathi, E. D. Emmons, J. A. Guicheteau, S. D. Christesen, P. G. Wilcox, D. K. Emge, A. W. Fountain, Trace Explosive Detection in Fingerprints with Raman Chemical Imaging, Proc. SPIE 7665 (2010) 76650N-1-76650N-6.

[33] A. Tripathi, E. D. Emmons, P. G. Wilcox, J. A. Guicheteau, D. K. Emge, S. D. Christesen, A. W. Fountain, Semi-Automated Detection of Trace Explosives in Fingerprints on Strongly Interfering Surfaces with Raman Chemical Imaging, Appl. Spectrosc. 65 (2011) 611-619.

[34] I. Malka, A. Petrushansky, S. Rosenwaks, I. Bar, Detection of explosives and latent fingerprint residues utilizing laser pointer-based Raman spectroscopy, Appl. Phys. B 113 (2013) $511-518$.

[35] Determinación de nitrógeno nítrico y NO3, in: Laboratorio Químico Central de Armamento, ENAC. Retrieved from http://www.enac.es/web/enac/acreditados

[36] M. López-López, J. L. Ferrando, C. García-Ruiz, Dynamite analysis by Raman spectroscopy as a unique analytical tool, Anal. Chem. 85 (2013) 2595-2600. 